\title{
Energy spectrum of the quantum vortices configurations
}

\author{
Sergey K. Nemirovskii \\ Institute of Thermophysics, 1 Lavrentyev Ave., Novosibirsk 630090, Russia \\ Novosibirsk State University, Novosibirsk, Russia \\ E-mail: nemir@itp.nsc.ru
}

Received September 7, 2014, published online April 23, 2015

\begin{abstract}
The energy spectra of the 3D velocity field, induced by various vortex filaments configurations are reviewed. The especial attention is paid to configurations generating the Kolmogorov type energy spectrum $E(k) \propto k^{-5 / 3}$. The motivation of this work is related to the problem of modeling classical turbulence with a set of chaotic vortex filaments. The quantity $<\mathbf{v}(\mathbf{k}) \mathbf{v}(-k)>$ can be exactly calculated, provided that we know the probability distribution functional $\mathcal{P}(\{\mathbf{s}(\xi, t)\})$ of vortex loops configurations. The knowledge of $\mathcal{P}(\{\mathbf{s}(\xi, t)\})$ is identical to the full solution of the problem of quantum turbulence and, in general, $\mathcal{P}$ is unknown. One of the simplifications is to investigate various truthful vortex configurations which can be elements of real vortex tangles. These configurations are: the uniform and nonuniform vortex arrays, the straight lines with excited Kelvin waves on it and the reconnecting vortex filaments. We demonstrate that the spectra $E(k)$, generated by the these configurations, are close to the Kolmogorov dependence $\propto k^{-5 / 3}$, and discuss the reason for this as well as the reason for deviation.

PACS: 67.25.dk Vortices and turbulence;

47.37.+q Hydrodynamic aspects of superfluidity; quantum fluids;

03.75.Kk Dynamic properties of condensates; collective and hydrodynamic excitations, superfluid flow.
\end{abstract}

Keywords: superfluidity, vortices, quantum turbulence.

\section{Introduction and scientific background}

The energy spectra of the 3D velocity field produced by various configurations of the quantized vortex filaments are described. Especial attention is paid to configurations generating the Kolmogorov type energy spectrum $E(k) \propto k^{-5 / 3}$. Such statement of problem is motivated by the problem of modeling classical turbulence with a set of chaotic vortex filaments. This idea has been discussed for quite a long time.(For details see, e.g., [1-3]). In classical fluids thin vortex tubes do not exist because they spread due to viscosity, so the concept of vortex filaments should just be considered as a model. Quantum fluids, where the vortex filaments are real objects, give an excellent opportunity for developing the study of the question of whether the dynamics of a set of vortex lines is able to reproduce (at least partially) the properties of real hydrodynamic turbulence. Among various arguments supporting the idea of quasiclassic behavior of quantum turbulence, the strongest is the $k$ dependence of the spectra of energy $E(k)$ obtained in numerical simulations and experiments. There are many works, that demonstrate a dependence of $E(k)$ close to the Kolmogorov law $E(k) \propto k^{-5 / 3}$. These are works, based on both the vortex filament method [4-6] and the Gross-
Pitaevskii equation $[7,8]$. The most common view of quasiclassical turbulence is the model of vortex bundles. The point is that the quantized vortices have a fixed core radius, so they do not possess the very important property of classical turbulence - stretching vortex tubes with a decrease in core size. The latter is responsible for the turbulence energy cascade from large scales to the small scales. Collections of near-parallel quantized vortices (vortex bundles) do possess this property, so the idea that the quasiclassical turbulence in quantum fluids is realized via vortex bundles of different sizes and intensities (number of threads) seems quite natural. Meanwhile, the concept of the bundle structure is vague and up to now it has not been definitely confirmed. It is unclear how the bundles can spontaneously appear (at low temperature, when the coupling with normal component is small). Moreover, even if they are prepared artificially, they are extremely unstable (see, $[3,9]$ ), can be easily destroyed in a result of reconnection either between the neighboring threads or in collisions with the other bundles, with the formation of the "bridging”. Therefore it quite tempting to find another alternative mechanism for the appearance of the Kolmogorov type spectrum, and we are looking for the various vortex filaments configurations as a candidate for this purpose. 
Of course, the more honest way is to resolve the general problem and find the vortex configuration studying the vortex dynamics on the basis of the motion equations. But this way is practically impossible due to the incredible complexity of the problem. In general, superfluid turbulence (ST) can be regarded as the kinetics of merging and splitting vortex loops, which, in addition, possess their own very involved dynamics, with an infinite number of degrees of freedom. We disregard for a time the presence of the very long lines stretching from wall to wall ("infinite lines" in the context of cosmic strings). Thus, in the full statement of the problem we have to deal with a set of objects (vortex loops), which does not have a fixed number of elements - the elements can be born and die. Thus, an analog of the secondary quantization method is required, with the difference being that the objects (vortex loops) themselves possess an infinite number of degrees of freedom with very involved dynamics. Clearly, this problem can hardly be resolved in the near future, and substantial simplifications are required. One of these simplifications is to investigate various truthful vortex configurations which can be elements of real vortex tangles.

In the work we introduce the general method for calculation of the energy spectrum via the vortex line configuration, then we consider the couple of simple but useful examples -the straight line and vortex ring of radius $R$. Then we study uniform and nonuniform vortex arrays, the straight line with excited Kelvin wave on it and then we study the case of the reconnecting vortex filaments. We demonstrate that the spectra $E(k)$, generated by the these configurations, is very close to the Kolmogorov dependence $\propto k^{-5 / 3}$, and discuss the reason for this as well as the reason for deviation.

\section{Calculation of spectrum}

Let us discuss the energy of the flow created by a vortex structure [10-12]. The energy of the vortex tangle is defined as

$$
E=\frac{1}{2} \int \rho_{s} \mathbf{v}_{s}^{2} d^{3} \mathbf{r}=\frac{\rho_{s} \kappa^{2}}{8 \pi} \sum_{j, i} \int_{0}^{L_{i}} \int_{0}^{L_{j}} \frac{\mathbf{s}_{i}^{\prime}\left(\xi_{i}\right) \cdot \mathbf{s}_{j}^{\prime}\left(\xi_{j}\right)}{\left|\mathbf{s}_{i}\left(\xi_{i}\right)-\mathbf{s}_{j}\left(\xi_{j}\right)\right|} d \xi_{i} d \xi_{j}
$$

Here we used that formally the vorticity field $\omega(r)$ can be written as

$$
\boldsymbol{\omega}(\mathbf{r})=\nabla \times \mathbf{v}_{s}=\kappa \int \mathbf{s}^{\prime}(\xi) \delta(\mathbf{r}-\mathbf{s}(\xi, t)) d \xi .
$$

In relations (1)-(2) we used $\mathbf{s}(\xi)=\bigcup \mathbf{s}_{i}\left(\xi_{i}\right)$ is the union of lines $\mathbf{s}_{i}\left(\xi_{i}\right)$ where $\mathbf{s}_{i}\left(\xi_{i}\right)$ describes the $i$-vortex line position parameterized by the label variable $\xi_{i}, s_{i}^{\prime}\left(\xi_{i}\right)$ denotes the derivative with respect to variable $\xi_{i}$ (the tangent vector) and $\int_{C}=\int_{C} \sum_{j}$.
By the use of the formula from the theory of generalized functions (see [13])

$$
\frac{1}{|\mathbf{r}|}=\int_{\mathbf{k}} \frac{d^{3} \mathbf{k}}{2 \pi^{2} k^{2}} \mathrm{e}^{i \mathbf{k r}},
$$

the average energy $E$ (1) can be rewritten as

$$
\begin{gathered}
E=\int_{\mathbf{k}} \frac{d^{3} \mathbf{k}}{(2 \pi)^{3}} \times \\
\times\left[\frac{1}{k^{2}} \frac{\rho_{s} \kappa^{2}}{2} \sum_{i, j} \int_{0}^{L_{i}} \int_{0}^{L} \mathbf{s}_{j}^{\prime}\left(\xi_{i}\right) \cdot \mathbf{s}_{j}^{\prime}\left(\xi_{j}\right) d \xi_{i} d \xi_{j} \mathrm{e}^{i \mathbf{k}\left(\mathbf{s}_{i}\left(\xi_{i}\right)-\mathbf{s}_{j}\left(\xi_{j}\right)\right)}\right] .
\end{gathered}
$$

Equation (4) is of crucial importance, since it offers a simple way of calculating the energy spectrum $E(\mathbf{k})$ of the three-dimensional velocity field induced by the vortex tangle. According to Eq. (4), the quantity $E(\mathbf{k})$ is just the expression inside the brackets. Note that the energy spectrum $E(\mathbf{k})$ can be also derived from the expression for the Fourier transform of the velocity field $v_{\mathbf{k}}=\mathbf{k} \times \omega_{\mathbf{k}} / \mathbf{k}^{2}$. Taking into account that due to incomressibility $\mathbf{k} \omega_{\mathbf{k}}=0$, one easily obtains $v_{\mathbf{k}} \cdot v_{-\mathbf{k}}=\omega_{\mathbf{k}} \cdot \omega_{-\mathbf{k}} / k^{2}$.

For the isotropic case, the spectral density depends on the absolute value of the wave number $k$. Integrating over solid angle lead to formula (see [14]):

$$
\begin{gathered}
E(k)=\frac{\rho_{s} \kappa^{2}}{(2 \pi)^{2}} \times \\
\times \sum_{i, j} \int_{0}^{L_{i}} \int_{0}^{L_{j}} \mathbf{s}_{j}^{\prime}\left(\xi_{i}\right) \cdot \mathbf{s}_{j}^{\prime}\left(\xi_{j}\right) d \xi_{i} d \xi_{j} \frac{\sin \left(k\left|\mathbf{s}\left(\xi_{i}\right)-\mathbf{s}\left(\xi_{j}\right)\right|\right)}{k\left|\mathbf{s}\left(\xi_{i}\right)-\mathbf{s}\left(\xi_{j}\right)\right|} .
\end{gathered}
$$

For anisotropic situations, formula (5) is understood as the angular average. Thus, for calculation of the energy spectrum $E(k)$ of the 3D velocity field, induced by the collapsing vortex filament we need to know the exact configuration $\{s(\xi)\}$ of vortex lines. Further we will apply these formulas to study some particular configurations $\{s(\xi)\}$ which probably induce the Kolmogorov spectrum $E(k) \propto k^{-5 / 3}$. Before we do it, let's describe the couple of simple but useful examples — the straight line and vortex ring of radius $R$.

The angle averaged spectrum created by straight vortex line is directly evaluated from (5) to give result $E_{\text {straight }}(k)=\left(\rho_{s} \kappa^{2} / 4 \pi\right) k^{-1}$ (per unit length). This spectrum is discussed early (see, e.g., $[7,15]$ proposed the $k^{-1}$ spectrum on the basis of dimensional consideration. This result is important, since it states that for any vortex system the high wave numbers larger than inverse curvature, $E(k)$ should scale as $k^{-1}$.

Now we will consider a vortex ring with radius $R$ lying in the $x-y$ plane. The line $s(\xi)$ can be parameterized as 


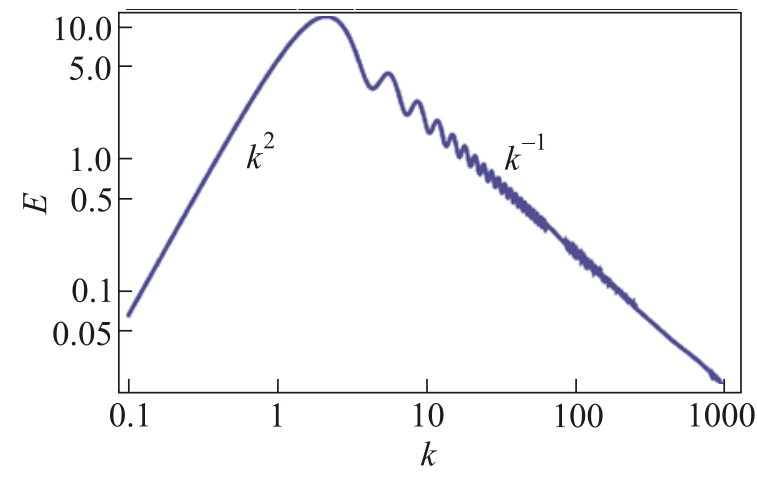

Fig. 1. (Color online) The spectrum induced by ring of unit radius (see text for explanations).

$s(x)=(R \cos \varphi, R \sin \varphi, 0)$ with $\varphi \in[0,2 \pi]$. Applying it to Eq. (5) we get (see also [7])

$$
\begin{gathered}
E_{\text {ring }}=\frac{\rho_{s} \kappa^{2} R}{(2 \pi)^{2}} \times \\
\times \int_{0}^{2 \pi 2 \pi} d \varphi_{0} d \varphi_{2} \frac{\cos \left(\varphi_{1}-\varphi_{2}\right) \sin \left(2 k R \sin \left(\left(\varphi_{1}-\varphi_{2}\right) / 2\right)\right)}{2 k \sin \left(\left(\varphi_{1}-\varphi_{2}\right) / 2\right)} .
\end{gathered}
$$

Evaluating the integral numerically shows that the spectrum $E_{\text {ring }}(k)$ scales like $E_{\text {ring }}(k) \sim k^{2}$ for $k R<<1$. Frequently, the $k^{2}$ is referred to as a proof for the thermodynamical equilibrium state. We would like to stress, however, that this distribution of the energy (valid far from the ring) has nothing to do with the equipartition law. It is a consequence of the fact that closed vortex loops induce a far field flow scaling as $1 / r^{3}$ (Fig. 1). That, in turn, generates a spectrum $E(k) \propto k^{2}$. This fact was established for classical turbulence (see, e.g., [18]). For quantum turbulence this result was discussed by Stalp, Skrbek and Donnelly [19]. For the large $k$, namely for $k R>>1$, spectrum $E_{\text {ring }}(k)$ scales like $\sim k^{-1}$ as for straight line.

\section{Vortex array}

\subsection{Uniform lattice}

In Introduction we discussed, that one of ways to support the quasi-classic behavior of quantum turbulence is the vortex bundles structure. Let's study what is the energy spectrum of 3D flow induced by the array of firtex filaments, imitating the bundle. First we consider a set of straight vortex filaments forming the square lattice $\bigcup s_{i}(\xi)=\bigcup\left(x_{i}, y_{j}, z\right)$. Points $x_{i}, y_{j}$ are coordinates for vortices on the $x y$-plane, indices $i, j$ runs from 1 to $N$. The neighboring lines are separated by distance $b$, i.e., $x_{i+1}-x_{i}=b$. In case of different straight lines we have to perform the integration between different lines and

$$
\begin{gathered}
\left|\mathbf{s}_{1}\left(\xi_{1}\right)-\mathbf{s}_{2}\left(\xi_{2}\right)\right|=\sqrt{\left(x_{1 i}-x_{2 i}\right)^{2}+\left(y_{1 j}-y_{2 j}\right)^{2}+\left(z_{i}-z_{j}\right)^{2}}= \\
=\sqrt{d_{i j}^{2}+\left(z_{i}-z_{j}\right)^{2}}
\end{gathered}
$$

where $d_{i j}=\sqrt{\left(x_{1 i}-x_{2 i}\right)^{2}+\left(y_{1 j}-y_{2 j}\right)^{2}}$ distances between vortices on the $x y$-plane. Then Eq. (5) can be rewritten as

$$
\frac{E(k)}{\rho_{s} \kappa^{2} L}=
$$

$$
=\frac{1}{4 \pi k} \sum_{i_{1}, i_{2}=1}^{N} \sum_{j_{1}, j_{2}}^{N} \int_{1}^{L} \int_{0}^{L} \frac{\sin \left(k \sqrt{d_{i j}^{2}+\left(z_{i}-z_{j}\right)^{2}}\right)}{\left(\sqrt{d_{i j}^{2}+\left(z_{i}-z_{j}\right)^{2}}\right)} d\left(z_{1}-z_{2}\right) .
$$

Integral over $z$ is in the table by Ryzhik-Gradshtein ((3.876), see [20])

$$
\frac{E(k)}{\rho_{s} \kappa^{2} L}=\frac{1}{4 \pi k} \sum_{i_{1}, i_{2}=1}^{N} \sum_{j_{1}, j_{2}=1}^{N} J_{0}\left(k d_{i j}\right) .
$$

Thus, determination of the spectrum on the basis (7) should be done with the use of the quadruple summation (over $\left(x_{i}, x_{j}, y_{i}, y_{j}\right)$ ), which requires large computing resources. Clear, however, that for very small $k$, which corresponds to very large distance, the whole array can be considered as large single vortex with the circulation $N^{2} \kappa$. Accordingly, the spectrum (per unit height) should be $\left(\rho_{s} N^{4} \kappa^{2} / 4 \pi\right) k^{-1}$. For large $k$, which corresponds to very small distance from each line, the spectrum (per unit height) should be $\left(\rho_{s} \kappa^{2} / 4 \pi\right) k^{-1}$. In the intermediate region $k b<<1$, and $N k b>>1$ (this condition implies that inverse wave number $k^{-1}$ is larger intervortex space between neighboring lines, but smaller then the size of the whole array $\mathrm{Nb}$ ), we can replace the quadruple summation by the quadruple integration with infinite limits. This procedure corresponds that we exclude the fine-scale motion near each of vortex, and are interested in the only largescale, coarse-grained motion. After obvious change of variables $x_{i} \rightarrow k x_{i}, y_{i} \rightarrow k y_{i}$ etc. we get that the whole integral should scale as $1 / k^{4}$, and accordingly $E(k) \propto 1 / k^{5}$ (compare with [17], Appendix A.3 and Subsection III.a ).

The obtained result can be illustrated in the 2D case. Noting that the full $E=\int E(k) d k=\int E(\mathbf{k}) d^{2} \mathbf{k}$, and in the isotropic case, it is $\int E(\mathbf{k}) 2 \pi k d k$. This implies that $E(\mathbf{k}) 2 \pi k=E(k), \quad$ and, correspondingly, $\quad E(\mathbf{k})=d E / d^{2} \mathbf{k}$ should behave as $1 / k^{-6}$. Since, further, $E(\mathbf{k})=\mathbf{v}(\mathbf{k}) \mathbf{v}(-\mathbf{k})$, we see that $v(\mathbf{k})$ scales as $1 / k^{-3}$. The latter means (see, e.g., [2], Eqs. (4.60), (4.61)) that the velocity $v(\mathbf{r})$ scales as $\mathbf{r}^{1}$. Indeed, in the $2 \mathrm{D}$ case we have the following chain of relations 


$$
\begin{gathered}
\mathbf{v}(\mathbf{r})=\frac{1}{2 \pi} \int \mathbf{v}(\mathbf{k}) \mathrm{e}^{i \mathbf{k r}} d^{2} \mathbf{k} \propto \frac{1}{2 \pi} \int \frac{1}{k^{3}} \mathrm{e}^{i k r \cos \theta} k d k d \theta= \\
=\int \frac{1}{k^{3}} J_{0}(k r) k d k .
\end{gathered}
$$

Thus, the velocity field $\mathbf{v}(\mathbf{r})$ scales with distance $r$ according to the following rule:

$$
\mathbf{v}(\mathbf{r}) \propto r \int \frac{1}{(k r)^{2}} J_{0}(k r) d(k r) .
$$

Thus, the uniform vortex array creates the coursegrained motion, which is rotation (velocity is proprtional to the distance from center), as it should be. Moreover, the coefficient is proportional to $\kappa / 2 b^{2}$, which corresponds to the Feynman rule. Integral in (9) does not converge, since it corresponds situation when all infinite plain rotates, but we have to take into account that this consideration (in addition approximate) concerns the domain inside of the vortex array and we ignorred all boundary effects. Concluding this subsection we state that the uniform vortex bundles do nt generate the Kolmogorov spectra.

\subsection{Noninform lattice}

Let's now consider the nonuniform vortex bundle. To model this situation we just can choose that the distance $b$ between lattice points (see previous subsection) is not constant, but depends on the numbers $i, j$ of the cell nodes. We have to realize that the problem of the spontaneous formation of vortex bundles is only son the stage of discussion so far, and there is no ideas concerning the arrangement of these bundles. We will choose the power law dependence for the distance between the lattice points.

$$
x_{i+1}-x_{i}=b_{0} i^{\lambda}, y_{j+1}-y_{j}=b_{0} j^{\lambda} \text {. }
$$

We do not ascertain the quantaty $\lambda$ it is free parameter of our approach. Under condition (10) the expression (7) turns into

$$
\frac{E(k)}{\rho_{s} \kappa^{2} L}=\frac{1}{4 \pi k} \sum_{i=1}^{N} \sum_{j=1}^{N} J_{0}\left(k b_{0} i^{\lambda}\right) .
$$

That means that the change the summation by integration we have to take $x_{i} \rightarrow k^{1 / \lambda} x_{i}, y_{i} \rightarrow k^{1 / \lambda} y_{i}$ (instead of the change of variables $x_{i} \rightarrow k x_{i}, y_{i} \rightarrow k y_{i}$ made previous subsection), etc. we get that the whole integral should scale as $1 / k^{1+4 / \lambda}$. It is easy to see that for $\lambda=6$, the spectrum $E(k) \propto k^{-5 / 3}$.
Currently, in classic hydrodynamics, the highly important topic - the role of hydrodynamic collapse and its role in the formation of turbulent spectra. is being intensively discussed (see, e.g., [21,22]). Briefly this phenomena can be described as spontaneous infinite grows of vorticity with formation of singularity $\omega(r)$. In particular, in the continuously distributed vortex field the vortex lines (not quantized vortex filaments, just hydrodynamic vortex lines!) approach each other and accumulates at some points forming singular distribution $\omega(r) \sim r^{-2 / 3}$. The latter results in the increment for velocity field $\langle\mathbf{v}(\mathbf{r}+\boldsymbol{\delta} \mathbf{r})\rangle \sim r^{1 / 3}$, which, in turn results it to the Kolmogorov spectrum $E(k) \propto k^{-5 / 3}$.

Let's find $2 D$ density of vortices on the xy plane under condition (10), or, according to the Feynman rule, the distribution of vorticity $\omega(r)$. In the "space" of indices $\{i, j\}$ vortices are distributed uniformly (one vortex per lattice site $\{i, j\}$ ), but since the distances between the sites vary, the distribution of vortices in the real $x y$ space is nonuniform. Let us consider "the ring" of radius from $I$ to $I+\Delta I$ in $\{i, j\}$. Then, the number of points $\Delta N$ in ring is just $2 \pi I \Delta I$, the radius of ring in real $x y$ space is $r=b_{0} I^{\lambda}$, and the thickness of ring is $\Delta r=b_{0} \lambda I^{\lambda-1} \Delta I$. From these relations it follows that $n(r)$ scales with $r$ as

$$
n(r)=\frac{\Delta N}{\Delta r} \propto \frac{1}{r^{1-2 / \lambda}} .
$$

If $\lambda=6$, then $n(r)=\omega(r)=r^{-2 / 3}$, as it should be for the classical turbulence [21].

\section{1D Kelvin waves spectrum and $3 D$ velocity spectrum}

In the literature there is discussed the idea of obtaining the $3 \mathrm{D}$ velocity spectrum just by putting it equal to the spectrum of 1D Kelvin waves. For instance, as stated in [23]. "We notice that, because the fluctuations of the velocity field are induced by the Kelvin wave fluctuations on the filaments, it is reasonable to expect that

$$
E(k) \sim E_{K W}(k), "
$$

The same idea was used in papers by Nazarenko et al. (see, e.g., [24]). Details of this activity can be read in a series of papers by L'vov, Nazarenko and coauthors [8,24-27].

Let us consider this problem on the basis of general formula (5). We take $\mathbf{s}(\xi, t)=(x(z, t), y(z, t), z)$ and denote the two-dimensional vector $(x(z, t), y(z, t))$ as $a \rho(z, t)$ (where the dimensionless amplitude $a<<1$ ). Substituting it into (5) and expanding in powers of $a$, we get,

$$
\begin{gathered}
E_{K W}(k)=E_{\text {straight }}(k)+a^{2} \frac{\rho_{s} \kappa^{2}}{(2 \pi)^{2}} \int_{00}^{L L} d z_{1} d z_{2}\left\{\frac{\cos \left(k\left|z_{2}-z_{1}\right|\right)\left(\rho\left(z_{2}\right)-\rho\left(z_{1}\right)\right)^{2}}{2\left|z_{2}-z_{1}\right|^{2}}-\right. \\
\left.-\frac{\sin \left(k\left|z_{2}-z_{1}\right|\right)\left(\rho\left(z_{2}\right)-\rho\left(z_{1}\right)\right)^{2}}{2 k\left|z_{2}-z_{1}\right|^{3}}+\frac{\left(\rho^{\prime}\left(z_{1}\right) \cdot \rho^{\prime}\left(z_{2}\right) \sin \left(k\left|z_{2}-z_{1}\right|\right)\right.}{k\left|z_{2}-z_{1}\right|}\right\} .
\end{gathered}
$$


To move further we have to find the correlation characteristics for the fluctuating vector of displacement $\rho\left(z_{2}\right)$. We accept that the ensemble of Kelvin waves has a following power-like spectrum:

$$
\langle\rho(p) \cdot \rho(-p)\rangle=A p^{-s} .
$$

We take here the notation $p$ for the one-dimensional vector, conjugated to $z$, reserving the notation $k$ for the absolute value of the wave vector of the $3 \mathrm{D}$ field. The formula (14) implies that (see, e.g., [2], Eqs. (4.60), (4.61)) the squared increment for the vector of displacement scales as, $\left\langle\left(\rho\left(z_{2}\right)-\rho\left(z_{1}\right)\right)^{2}\right\rangle \propto\left(z_{2}-z_{1}\right)^{s-1}$. Then the second order correlator $\left\langle\left(\rho^{\prime}\left(z_{2}\right) \rho^{\prime}\left(z_{1}\right)\right)\right\rangle$ scales as $\left\langle\left(\rho^{\prime}\left(z_{2}\right) \rho^{\prime}\left(z_{1}\right)\right)\right\rangle \propto\left(z_{2}-z_{1}\right)^{s-3}$. Substituting it into (13) and counting the powers of quantity $k$, we conclude that the correction $\delta E(k)$ to the spectrum $E(k)$, due to the ensemble of Kelvin waves has a form:

$$
\delta E(k) \propto a^{2} k^{-s+2} .
$$

It is remarkable fact that this quantity coincides formally with the one-dimensional spectrum of KW $\delta E(p) \propto a^{2} p^{-s+2}$ however this contribution is small, by virtue the smallness of the wave amplitudes $a$, and disappears with the KW.

In series papers L'vov, Nazarenko and coauthors [8,24-26] it was proposed the spectrum for Kelvin waves of shape (14) with $s=11 / 3$, therefore the 3D spectrum of velocity field $\delta E(k) \propto k^{-5 / 3}$, i.e., the Kolmogorov type energy spectrum.

\section{Reconnecting lines}

Let's now describe the energy spectrum of the $3 \mathrm{D}$ velocity field, induced by vortex filaments collapsing towards reconnection [28]. The first obstacle is the exact determination of the vortex lines configurations. Despite the huge number of works devoted to the dynamics of collapsing lines both in classic and quantum fluids [29,30] (this list is far from full) the exact solution $\mathbf{s}(\xi)$ for the shape of curves has not yet obtained. The main results are obtained by different approaches, combining analytical and numerical methods, such as the vortex filament method (in both the local induction approximation and the Biot-Savart law), as well as the full 3D studies of Navier-Stocks equations or the Nonlinear Schrödinger equation for vortices in the Bose-Einstein condensate.

Qualitatively, the results of these investigations are quite similar and can be described as follows. Due to long range interaction in the Biot-Savart integral, the initially arbitrarily oriented vortices, when they approach each other, start by reorienting their close segments so as to bring them into an antiparallel position. Further, cusps may appear on the approaching segments of two vortex lines. The curvature of these cusps may be so large that the selfinduced velocity of each perturbation overcomes the repul- sion from the adjoining vortex line. Further the cusps grow and approach each other closer; this increases their curvature and, correspondingly, their self-induced velocities and this process is repeated faster and faster. It is important that this process grows explosively, since the distance between the two perturbed segments, $\Delta$, decreases according to the relation $\Delta \sim\left(t^{*}-t\right)^{1 / 2}$, where $t^{*}$ is some quantity depending on the relevant parameters and initial conditions. Thus, in a finite time the vortex lines collapse. Asymptotic lines are two hyperbolic curves lying on opposite sides of the pyramid (see, e.g., [30,33]). However, in a recent study, [31] it was shown that the curves are not exact hyperbolas, but slightly different lines (the authors call these curves quasi-hyperbolae) of type

$$
h(\xi)=\sqrt{a^{2} \xi^{2} /\left(a^{2}+\xi^{2}\right)+a^{2}+\xi^{2}},
$$

and that they lie not in the planes of the pyramid sides but on the curved surfaces, bent inwards. In the moments just before the collapse, when the vortex cores are nearly touch each other, the very acute kink appears. This curves may be written in parametric form (cf. formula (16) of [31])

$$
\mathbf{s}_{1,2}(\xi)=[ \pm(h(\xi)-c), \pm \xi,(h(h(\xi)-b))] \text {. }
$$

The described configuration is shown in Fig. 2. The signs are chosen so that $\mathbf{s}_{1}^{\prime}(0) \cdot \mathbf{s}_{2}^{\prime}(0)=-1$ (the vortices are antiparallel). Quantity $a$ is of the order of the curvature radius on the tip of the kink of the curve; quantity $b$ (related to $a$, see [31]) is responsible for bending the surfaces on which the quasi-hyperbolae lie. Quantity $c$ is also of the order of $a$ and is responsible for closeness of the filaments. All three quantities are smaller than the intervortex space $\delta=\mathcal{L}^{-1 / 2}$ (where $\mathcal{L}$ is the vortex line density). This vision is consistent with the results of numerous numerical works, studying the collapse of vortex lines (see, e.g., $[22,23]$ and references therein, the decisive picture obtained in [32] is shown in the inset of Fig. 2).

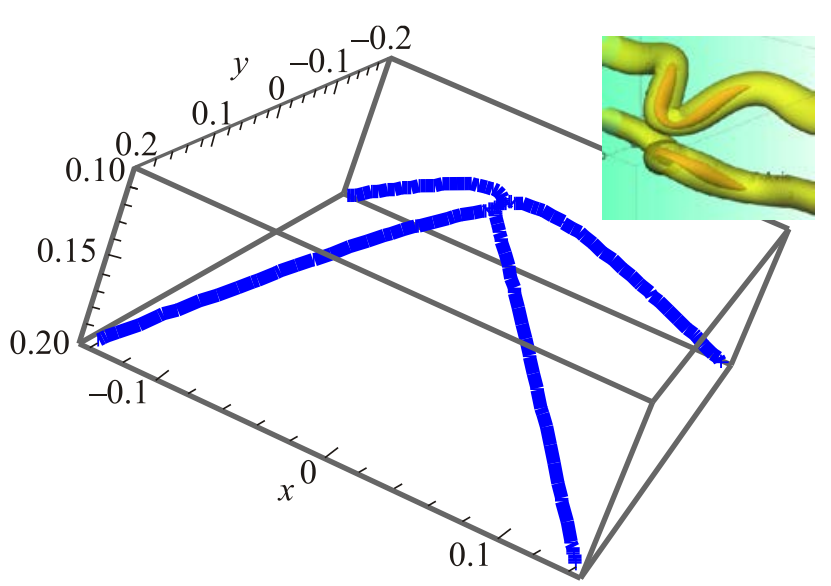

Fig. 2. (Color online) The touching quasi-hyperbolae describing the collapsing lines (see Eq. (16)) obtained in [31]. In the inset we set (as an example) the kinks on the anti-parallel collapsing vortex tubes obtained in numerical simulation [32]. 

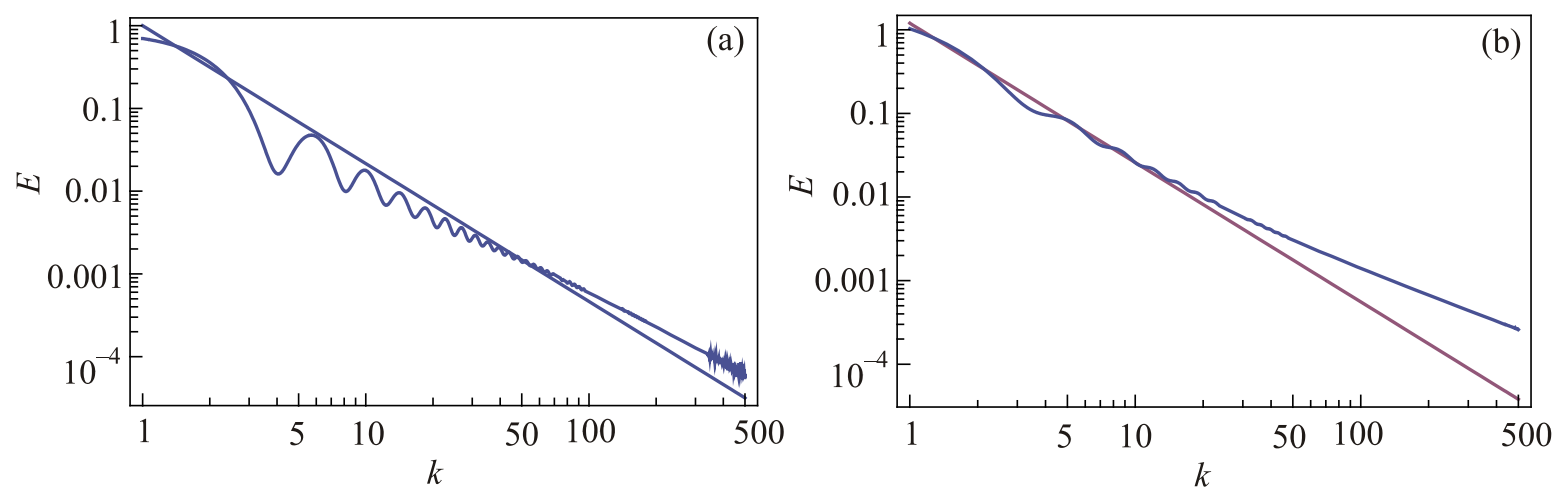

Fig. 3. (Color online) The same spectrum obtained on the basis of procedure described in the text (see Eq. (17)) (a). The spectrum $E(k)$, obtained numerically on the base formula (5). The straight line has a slope $-5 / 3$ (b).

In the Fig. 3(a) we presented the results of numerical calculation of spectrum $E(k)$ on the basis of formula (5) (without prefactor before integral) using a configuration $\{\mathbf{s}(\xi)\}$ of vortex lines described by (16). We chose the following parameters: $a=0.1, b=0.099, c=0.1$ (the case $a \approx c$ corresponds to nearly touching curves). It is seen that in the interval of wave numbers $k$ between 1-50 the slope of $E(k)$ is indeed close to $-5 / 3$. Because of the rapidly oscillating function, the evaluation of integral (5) is difficult, even numerically. In addition, numerical results obscure underlying physics. Therefore, to explain appearing of the Kolmogorov-type spectrum we performed an analytical study [28]. Referring the reader for details to the author's work [28], we briefly describe main speculations. The integral (5) can be approximately evaluated for large $k$ using the method of asymptotic expansion [34]. When $k$ is large the function $\sin \left(k\left|\mathbf{s}\left(\xi_{1}\right)-\mathbf{s}\left(\xi_{2}\right)\right|\right)$ is a rapidly varying function; therefore, the main contribution into integral comes from points of minimal value of the separation function between points of the curves $D\left(\xi_{1}, \xi_{2}\right)=$ $=\left|\mathbf{s}\left(\xi_{1}\right)-\mathbf{s}\left(\xi_{2}\right)\right|$. This is enhanced by the fact that the distance is included in the denominator in the integrand of (5).

Thus, the behavior of the phase function $D\left(\xi_{1}, \xi_{2}\right)$ near minimum is crucial for value of the integral and for its $k$ dependence. Let us study the phase function $D\left(\xi_{1}, \xi_{2}\right)$ for the vortex configuration described by Eq. (16) just before collapse when $c \approx a$. It is convenient to introduce variables $\rho=\xi_{1}-\xi_{2}$ and $R=\left(\xi_{1}+\xi_{2}\right) / 2$ and recast the double integral $\int_{C} \int_{C} d \xi_{1} d \xi_{2}$ as multiple integral $\int d R \int d \rho$ in the domain bounded by lines $\rho=2 R$ and $\rho=-2 R$. Analysing properly the phase function $D(\rho, R)$ and using of the methods of asymptotic expansion we can show that the spectrum $E(k)$ is

$$
E(k)=\left.\rho_{s} \kappa^{2} \int_{0} d R \sqrt{\frac{\pi}{k \partial^{2} D /\left.\partial \rho^{2}\right|_{\rho=0}}} \frac{\sin (k D(\rho, R))}{k D(\rho, R)}\right|_{\rho=0} .
$$

In the Fig. 3(b) we present $E(k)$, calculated on the basis of formula (17). First, please note, that spectrum calculated with the use of (17) very close to the spectrum calculated on basis (5); this justifies the approximated procedure, as described above. Second, and more importantly, the fact is that again in the interval of wave numbers $k$ between 1-50 the slope of $E(k)$ is close to $-5 / 3$. Analyzing the procedure one can see that the $\approx k^{-5 / 3}$ dependence appears because of in the most important interval of scale from the curvature of kinks to interline distance $\delta$ the quantity $D(0, R)$ is close to $R^{3 / 2}$. In fact, the authors of the numerical works [4-8] cited in the introduction, obtained the spectrum $E(k) \approx k^{-5 / 3}$ for the wave numbers $k$, around $k \approx 2 \pi / \delta$.

\section{Conclusions}

Summarizing, it can be concluded that the 3D energy spectrum $E(k)$ consists of several parts. At small $k$, associated with the large scales, on the order of the size of the system, or on the scale of the stirring forcing (grids, propellers, vibrating objects, etc.), the spectrum behaves as $E(k) \propto k^{2}$. We recall again that this is the consequence of the asymptotic behavior $(r \rightarrow \infty)$ of the velocity field, not of thermodynamical equilibrium. For large wave numbers, exceeding the inverse intervortex space $k>2 \pi / \delta$, the energy spectrum $E(k)$ should be close to $k^{-1}$, again regardless of the specific model. The region of intermediate $k$ is the most intriguing and exciting, it depends on the detailed structure of the vortex tangle. Coming back to the aims of the work stated in the introduction we can suggest that the spectrum $E(k)$ close to the Kolmogorov dependence $\propto k^{-5 / 3}$, which was observed in many numerical simulations on the dynamics of quantized vortex filaments [4-8], can appear from the some "standard" configurations composing the real vortex tangle.

The work was supported by the grant N 14-19-00352 from RSCF (Russian Scientific Foundation). 
1. A. Chorin, Vorticity and Turbulence, Applied Mathematical Sciences, Springer-Verlag (1994).

2. U. Frisch, Turbulence, Cambridge University Press, Cambridge (1995).

3. S.K. Nemirovskii, Phys. Rep. 524, 85 (2013).

4. T. Araki, M. Tsubota, and S.K. Nemirovskii, Phys. Rev. Lett. 89, 145301 (2002).

5. D. Kivotides, C.J. Vassilicos, D.C. Samuels, and C.F. Barenghi, Europhys. Lett. 57, 845 (2002).

6. D. Kivotides, C.F. Barenghi, and D.C. Samuels, Europhys. Lett. 54, 771 (2001).

7. C. Nore, M. Abid, and M.E. Brachet, Phys. Rev. Lett. 78, 3896 (1997).

8. N. Sasa, T. Kano, M. Machida, V.S. L’vov, O. Rudenko, and M. Tsubota, Phys. Rev. B 84, 054525 (2011).

9. G. Volovik, J. Exp. Theor. Phys. Lett. 78, 533 (2003).

10. S.K. Nemirovskii, Phys. Rev. B 57, 5972 (1998).

11. S.K. Nemirovskii, M. Tsubota, and T. Araki, J. Low Temp. Phys. 126, 1535 (2002).

12. S. Nemirovskii, J. Low Temp. Phys. 171, 504 (2013), ISSN 0022-2291 .

13. V.S. Vladimirov, Generalized Functions in Mathematical Physics (1976).

14. L. Kondaurova and S.K. Nemirovskii, J. Low Temp. Phys. 138, 555 (2005).

15. C. Nore, M. Abid, and M.E. Brachet, Phys. Fluids 9, 2644 (1997).

16. W.F. Vinen, Phys. Rev. B 61, 1410 (2000).

17. B. Nowak, J. Schole, D. Sexty, and T. Gasenzer, Phys. Rev. A 85, 043627 (2012).
18. A. Monin and A. Yaglom, Statistical Fluid Dynamics, MIT, Cambridge, MA (1975), Vol. II.

19. S.R. Stalp, L. Skrbek, and R.J. Donnelly, Phys. Rev. Lett. 82, 4831 (1999).

20. I.S. Gradshteyn and I.M. Ryzhik, Table of Integrals, Series, and Products, Fourth Academic Press (1980), Vol. 4.

21. E. Kuznetsov and V. Ruban, J. Exp. Theor. Phys. 91, 775 (2000).

22. R.M. Kerr, Phys. Fluids 25, 065101 (2013).

23. D. Kivotides, J.C. Vassilicos, D.C. Samuels, and C.F. Barenghi, Phys. Rev. Lett. 86, 3080 (2001).

24. V. L'vov, S. Nazarenko, and O. Rudenko, J. Low Temp. Phys. 153, 140 (2008).

25. V.S. L'vov, S.V. Nazarenko, and O. Rudenko, Phys. Rev. B 76, 024520 (2007).

26. L. Boué, R. Dasgupta, J. Laurie, V. L’vov, S. Nazarenko, and I. Procaccia, Phys. Rev. B 84, 064516 (2011).

27. S. Nemirovskii, J. Pakleza, and W. Poppe, Rus. J. Eng. Thermophys. 3, 369 (1993).

28. S.K. Nemirovskii, Phys. Rev. B 90, 104506 (2014).

29. E.D. Siggia, Phys. Fluids 28, 794 (1985).

30. R. Tebbs, A. Youd, and C. Barenghi, J. Low Temp. Phys. 162, 314 (2011).

31. L. Boué, D. Khomenko, V.S. L'vov, and I. Procaccia, Phys. Rev. Lett. 111, 145302 (2013).

32. M.D. Bustamante and R.M. Kerr, Physica D 237, 1912 (2008).

33. A.T.A.M. de Waele and R.G.K.M. Aarts, Phys. Rev. Lett. 72, 482 (1994).

34. M.V. Fedoryuk, Method of Saddle Points, Nauka, Moscow (1977). 